\title{
Physically Cross-Linked Double-Network Hydrogel for High-Performance Oil-Water Separation Mesh
}

Yong Liu,${ }^{\dagger}$ Meng Xia,${ }^{\dagger}$ Lili Wu,${ }^{\dagger}$ Shenxin Pan,${ }^{\dagger}$ Yuhong Zhang, $,{ }^{*},{ }^{\dagger}$ Benqiao He ${ }^{\dagger}$

\author{
Peixin $\mathrm{He},{ }^{*}{ }^{\dagger}$
}

${ }^{+}$Hubei Collaborative Innovation Center for Advanced Organic Chemical Materials, Ministry of

Education Key Laboratory for Green Preparation and Application of Functional Materials, Hubei University, Wuhan 430062, P. R. China

${ }^{\ddagger}$ State Key Laboratory of Separation Membranes and Membrane Processes, School of Materials Science and Engineering, Tianjin Polytechnic University, Tianjin 300387, P. R. China 

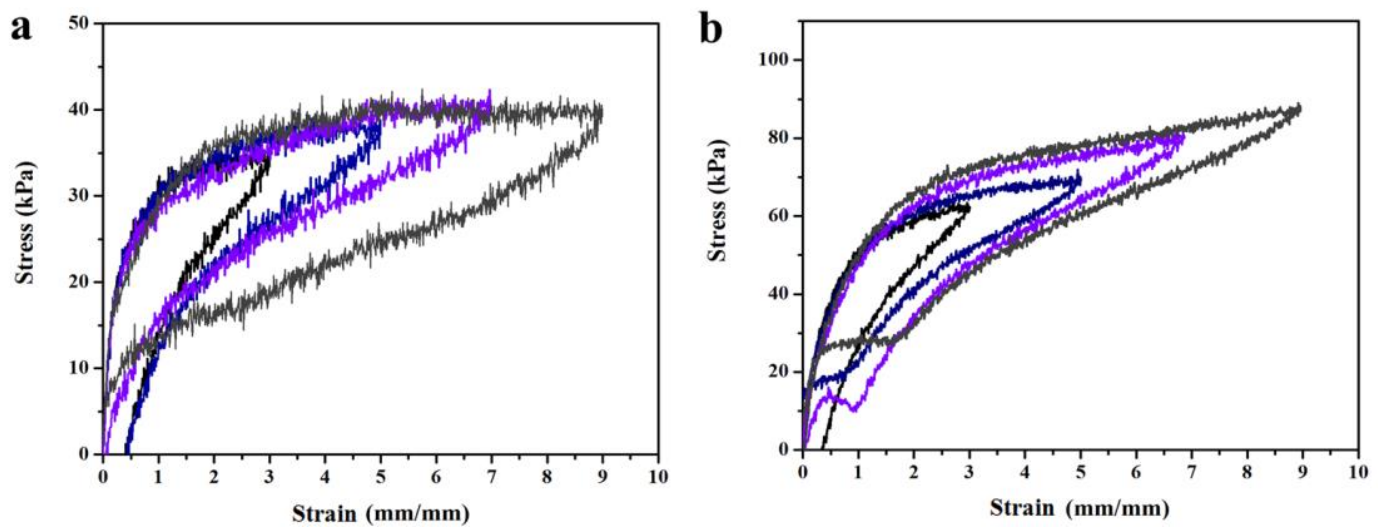

Figure S1. Tensile loading-unloading tests of (a) HPC-Gel and (b) EPC-Gel under different strains. 

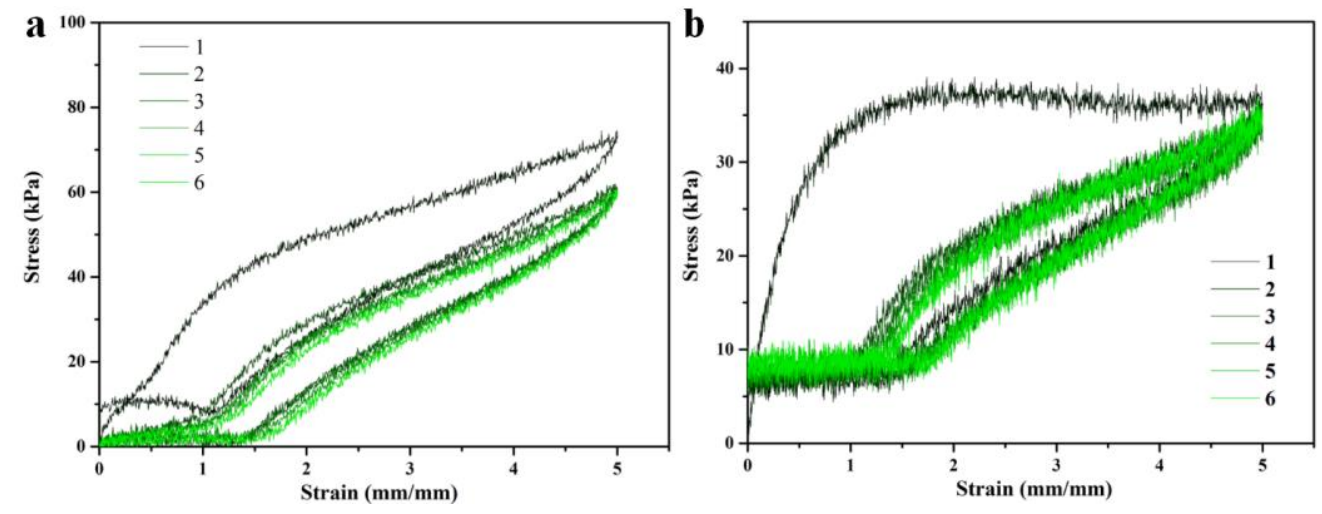

Figure S2. Six consecutive cycles of loading-unloading test for (a) EPC-Gel and (b) HPC-Gel. 


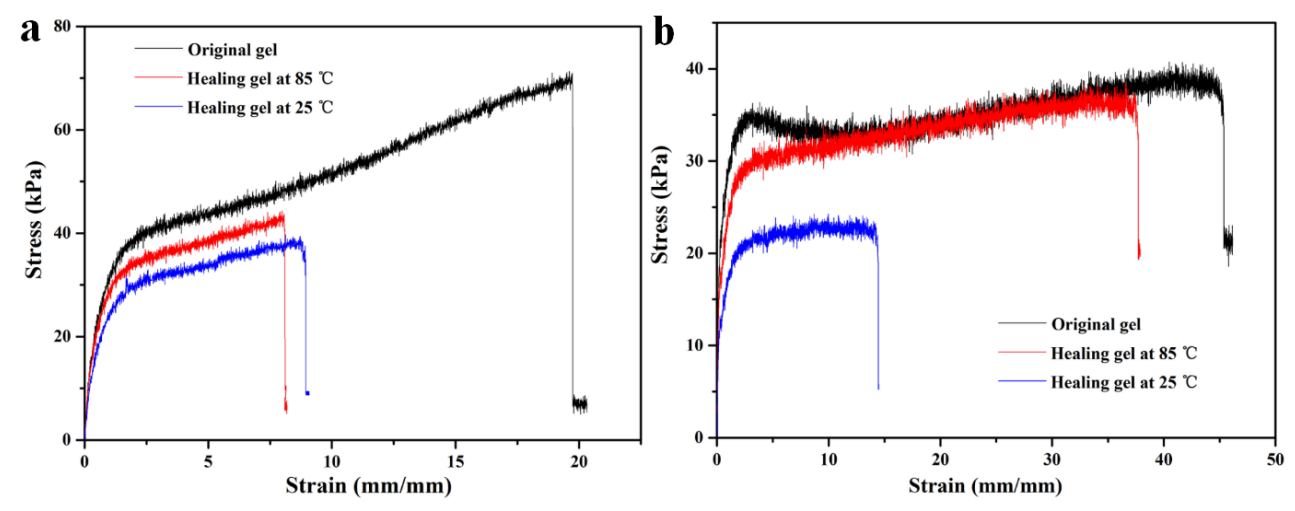

Figure S3. the self-healing capabilities of (a) EPC-Gel and (b) HPC-Gel at $25^{\circ} \mathrm{C}$ and $85{ }^{\circ} \mathrm{C}$. 

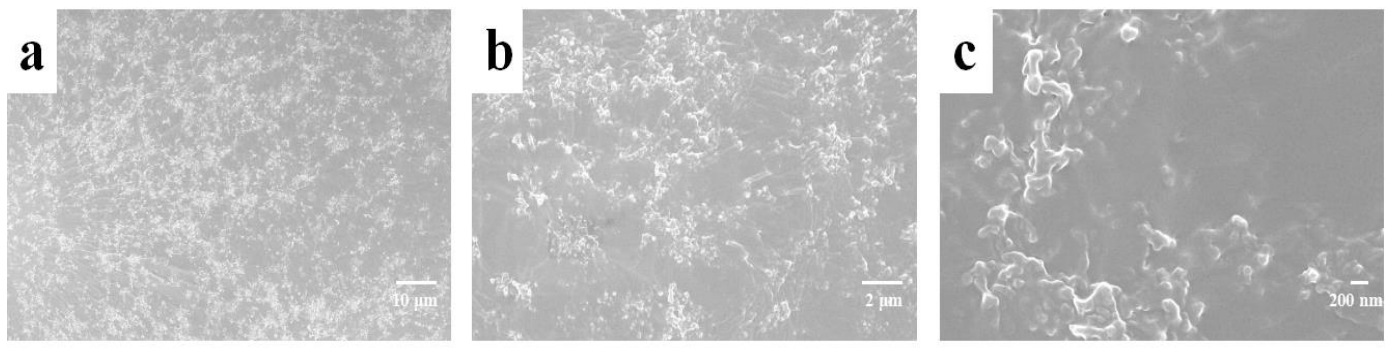

Figure S4. (a, b, c) SEM images of HEPC-Gels at three different magnifications. 


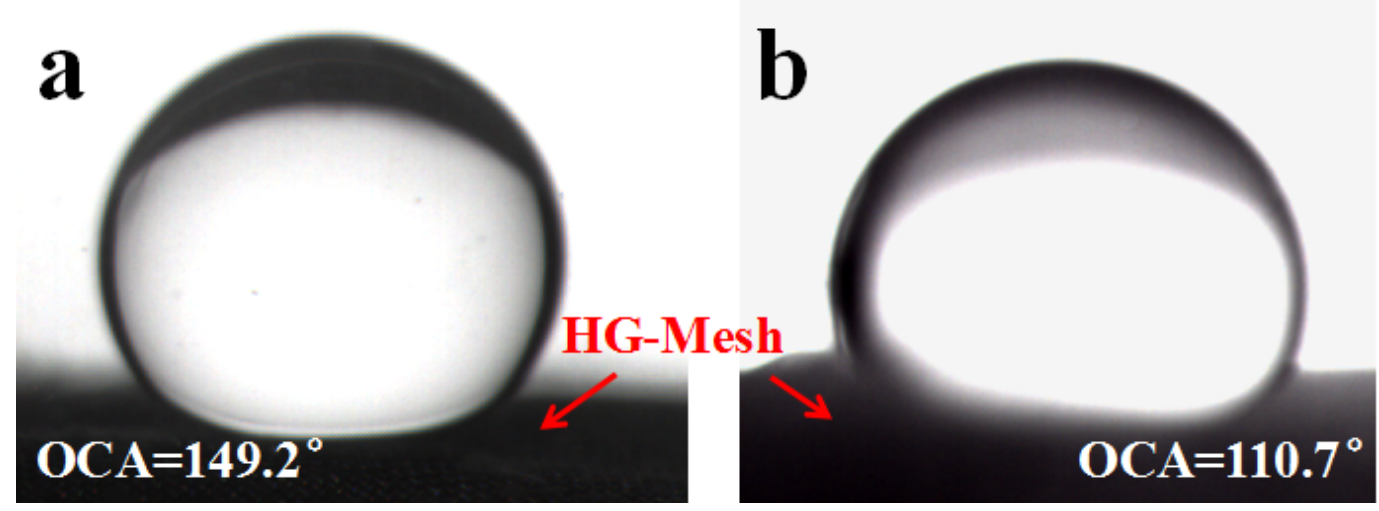

Figure S5. Underwater OCAs of the HG-Mesh immersed in water after (a) $0 \mathrm{~h}$ and (b) $18 \mathrm{~h}$. 


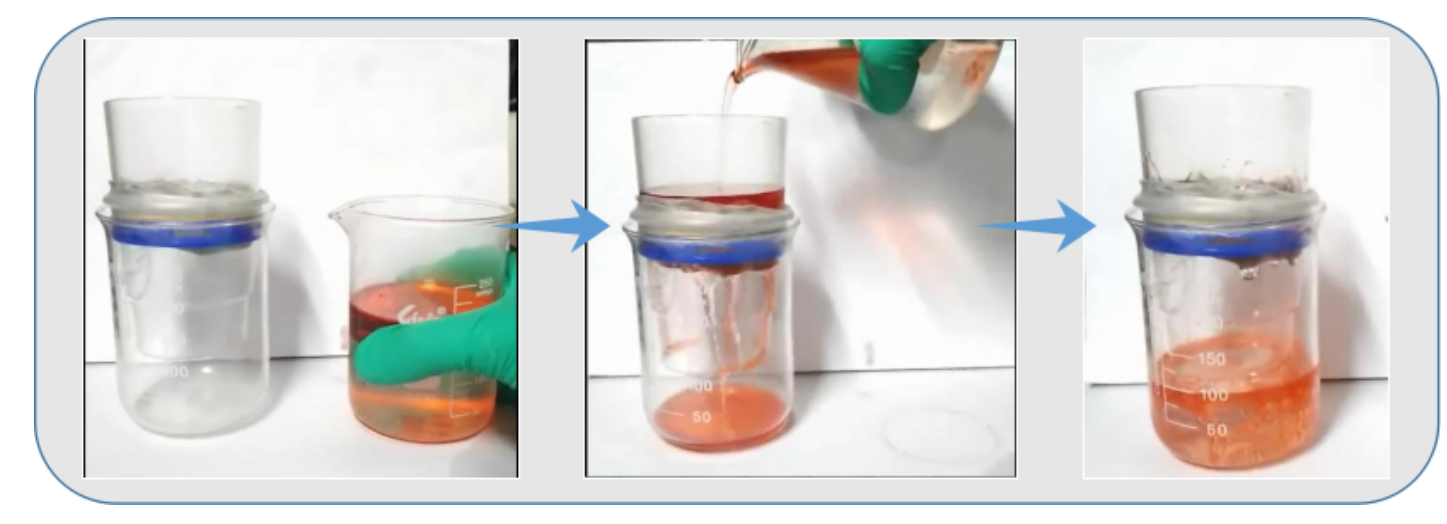

Figure S6. Separation process of water and cooking oil (red) on the naked mesh $\left(\rho_{\text {oil }}<\rho_{\text {water }}\right)$ 

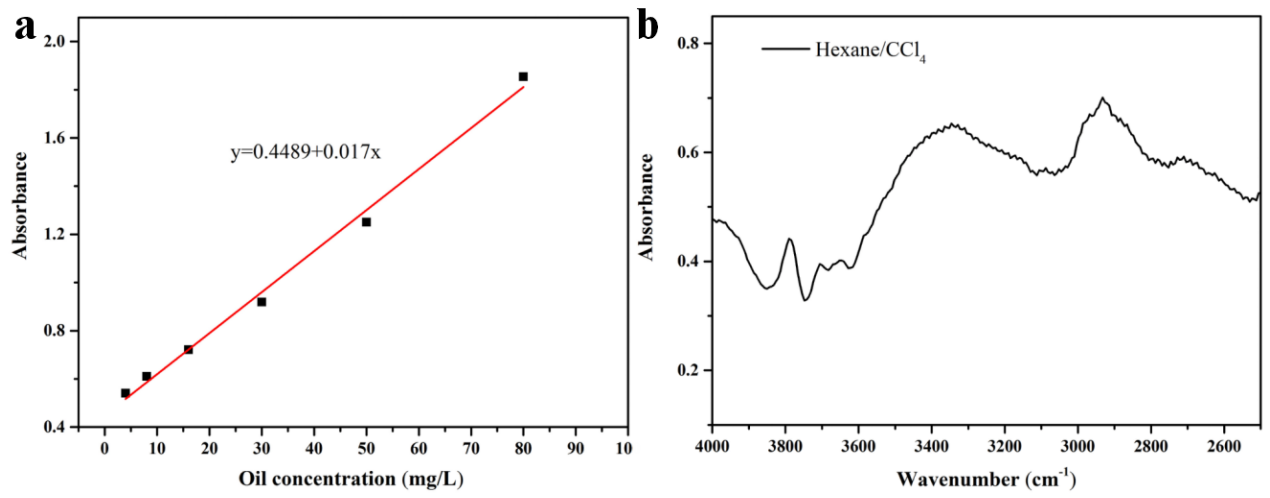

Figure S7. (a) The standard curve for different concentrations of hexane-in- $\mathrm{CCl}_{4}$ (b) Infrared spectrum of the extracted hexane/CCl $\mathrm{Cl}_{4}$ mixture. 


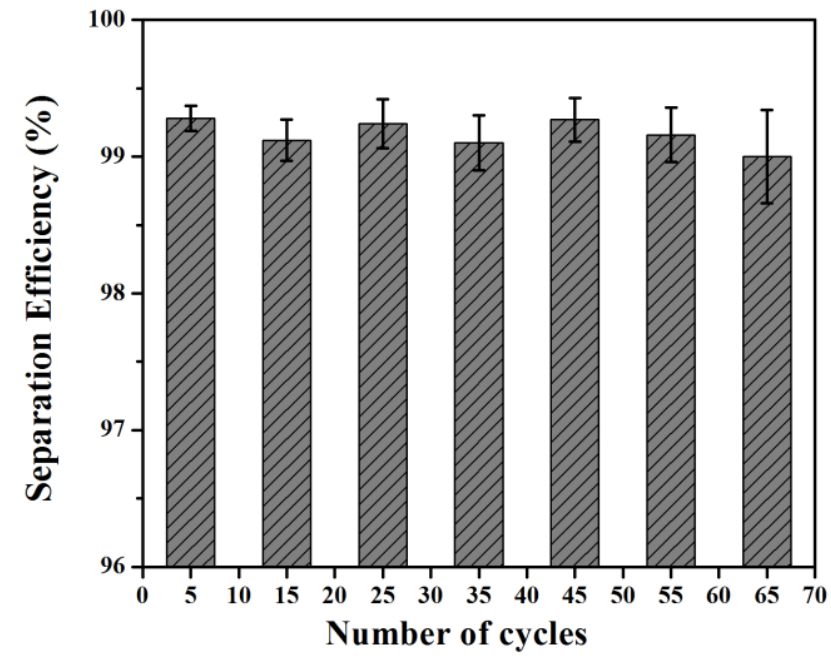

Figure S8. Separation efficiency at different cycle times for the mixture of cooking oil and water. $\left(\rho_{\text {oil }}<\rho_{\text {water }}\right.$ at $\left.25^{\circ} \mathrm{C}\right)$ 

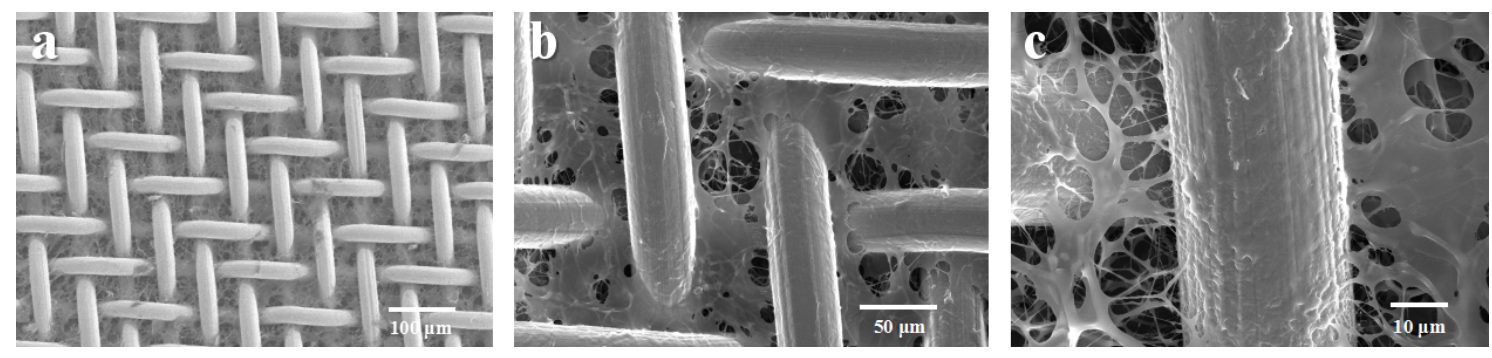

Figure S9. Typical SEM images of the wire meshes: $(a, b, c)$ Three different magnification images on HEG-Mesh after being recycled for 65 times. 

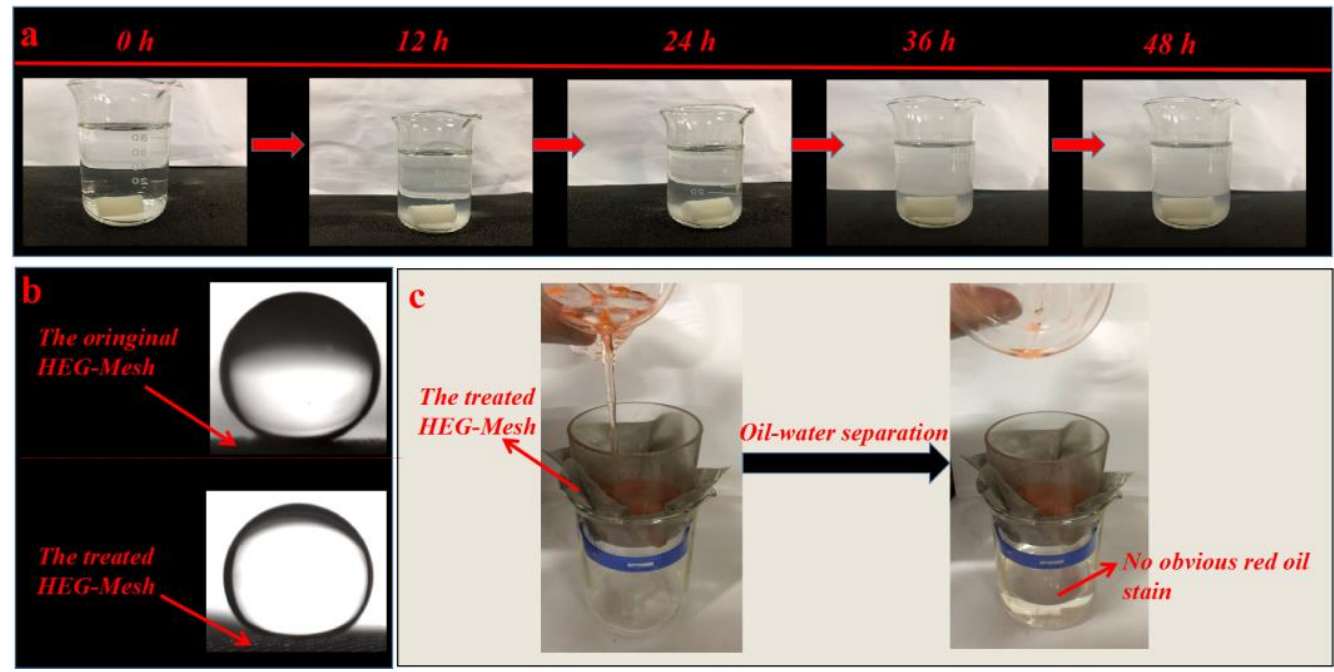

Figure S10. (a) Optical images of HEPC-Gel exposed to $2000 \mathrm{mg} / \mathrm{L}$ hypochlorite solution for

different times at $\mathrm{pH}=4.0$ and $25{ }^{\circ} \mathrm{C}$. (b) Optical images of underwater oil drops on the original HEG-Mesh and HEG-Mesh treated with hypochlorous acid solution for $12 \mathrm{~h}$. (c) Images show that oil-water separation ability of HEG-Mesh treated with hypochlorous acid solution for $12 \mathrm{~h}$. 


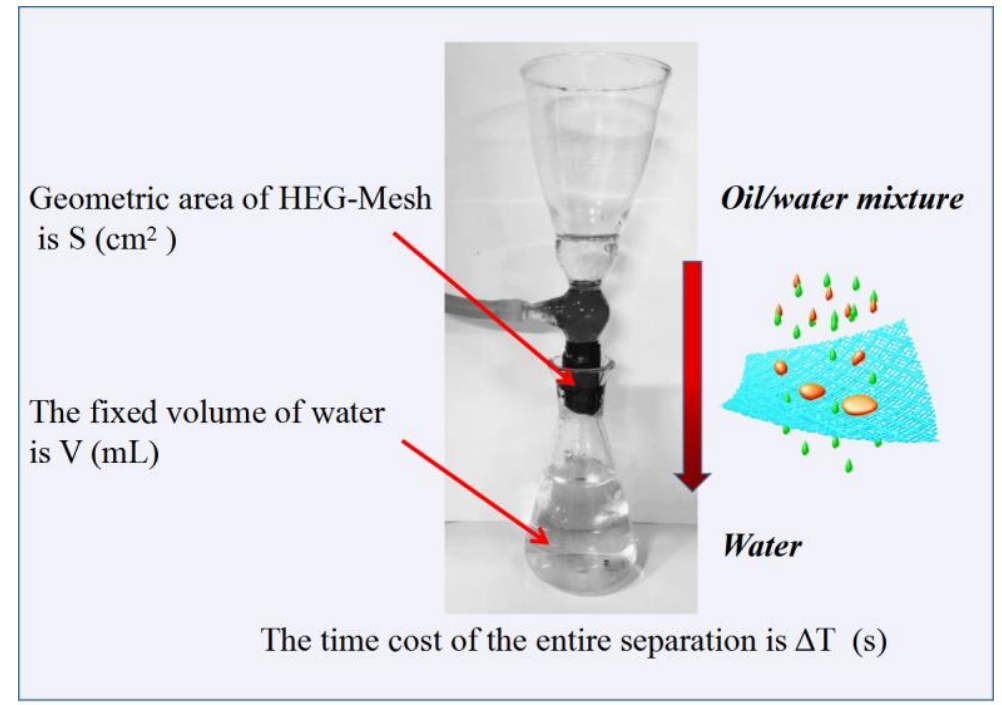

Figure S11. Schematic diagram and relevant data for testing water flux. 
Table S1. Comparison of the hydrogel-coated mesh based on mechanical property, separation efficiency, water flux, and tolerance to harsh environments.

\begin{tabular}{|c|c|c|c|c|c|}
\hline $\begin{array}{l}\text { Hydrogel (coated on the } \\
\text { mesh) }\end{array}$ & Mechanical property & $\begin{array}{l}\text { Separation } \\
\text { efficiency }\end{array}$ & $\begin{array}{l}\text { Water } \\
\text { flux }(\mathrm{L} \\
\left.\mathrm{m}^{-2} \mathrm{~h}^{-1}\right)\end{array}$ & Tolerance to harsh environments & Ref. \\
\hline $\begin{array}{l}\text { DKGM hydrogel on } \\
\text { glass fabric }\end{array}$ & $\begin{array}{c}110 \mathrm{MPa} \text { stress, } 4 \% \\
\text { strain }\end{array}$ & $>99.2 \%$ & 723.7 & $\begin{array}{c}\text { Physical abrasion durability, } \\
\text { corrosive organic solvents, salt } \\
\text { aqueous solution, acidic, alkaline } \\
\text { solutions }\end{array}$ & {$[\mathrm{S} 1]$} \\
\hline $\begin{array}{c}\text { Cellulose } \\
\text { hydrogel-coated mesh }\end{array}$ & - & $>98.9 \%$ & 12,885 & Salt aqueous solutions & {$[\mathrm{S} 2]$} \\
\hline $\begin{array}{l}\text { Guar gum-based } \\
\text { hydrogel-coated mesh }\end{array}$ & - & $>99.7 \%$ & 2850 & $\begin{array}{l}\text { Acidic, alkaline, or salt aqueous } \\
\text { solutions }\end{array}$ & {$[\mathrm{S} 3]$} \\
\hline Modifie Chitosan & & $>99 \%$ & - & $\begin{array}{c}\text { Acidic, alkaline, or salt aqueous } \\
\text { solutions }\end{array}$ & {$[\mathrm{S} 4]$} \\
\hline $\begin{array}{l}\text { DMAEMA-co-MAA } \\
\text { hydrogel }\end{array}$ & $\begin{array}{c}\text { Storage } \\
\text { modulus }>30000 \mathrm{~Pa}\end{array}$ & $>98.35 \%$ & - & $\mathrm{pH}$ and thermo sensitive & {$[\mathrm{S} 5]$} \\
\hline $\begin{array}{l}\text { PAAS-g-PVDF hydrogel } \\
\text { coated copper mesh }\end{array}$ & $\begin{array}{l}0.7 \text { MPa stress, } \\
200 \% \text { strain }\end{array}$ & $>97.1 \%$ & 50000 & $\begin{array}{l}\text { Anti-Crude-Oil-Adhesion, } \\
\text { acidic, or alkaline aqueous } \\
\text { solutions }\end{array}$ & [S6] \\
\hline $\begin{array}{c}\text { Cellulose } \\
\text { hydrogel-coated } \\
\text { colander }\end{array}$ & - & $>99.99 \%$ & - & Various oil phases & {$[\mathrm{S} 7]$} \\
\hline $\begin{array}{l}\text { calcium alginate } \\
\qquad \text { (Ca-Alg) }\end{array}$ & - & $>99.5 \%$ & 7800 & $\begin{array}{c}\text { Acidic, alkaline, or salt aqueous } \\
\text { solutions }\end{array}$ & {$[\mathrm{S} 7]$} \\
\hline $\begin{array}{c}\text { SIPN-Gels9:1 } \\
\text { hydrogel-coated mesh }\end{array}$ & $\begin{array}{c}\text { Rubber-like } \\
\text { mechanical property } \\
(57.3 \pm 2.4 \mathrm{kPa} \\
\text { stress, } 1540 \pm 55 \% \\
\text { strain) }\end{array}$ & $>99 \%$ & 8600 & $\begin{array}{l}\text { Acidic, alkaline, or salt aqueous } \\
\text { solutions, self-healing }\end{array}$ & {$[\mathrm{S} 8]$} \\
\hline $\mathrm{CH}_{8: 2}$ gel & $1300 \%$ elongation & $\begin{array}{l}\text { Above } \\
98.67 \%\end{array}$ & 8582 & $\begin{array}{l}\text { Acidic, alkaline, or salt aqueous } \\
\text { solutions,self-healing }\end{array}$ & [S9] \\
\hline
\end{tabular}



solutions

tensile stress of

$\sim 164 \mathrm{kPa}$, tensile

HEG-Mesh strain of $\sim 28$ $\mathrm{mm} / \mathrm{mm}$ and $>99 \%$ 11000

Acidic, alkaline, or salt aqueous present solutions, self-healing work fracture energy of $\sim 3.37 \mathrm{MJ} / \mathrm{m}^{2}$

\section{REFERENCES}

[S1] H. You, Y. Jin, J. Chen, C. Li. Chen, Direct coating of a DKGM hydrogel on glass fabric for multifunctional oilwater separation in harsh environments, Chem. Eng. J. 334 (2018) 2273-2282.

[S2] C. Ao, R. Hu, J. Zhao, X. Zhang, Q. Li, T. Xia, W. Zhang, C. Lu, Reusable, salt-tolerant and superhydrophilic cellulose hydrogel-coated mesh for efficient gravity-driven oil/water separation, Chem. Eng. J. 338 (2018): 271-277.

[S3] L. Zang, J. Ma, D. Lv, Q. Liu, W. Jiao, P. Wang, A core-shell fiber-constructed pH-responsive nanofibrous hydrogel membrane for efficient oil/water separation, J. Mater. Chem. A 5 (2017) 19398-19405.

[S4] S. Zhang, F. Lu, L. Tao, N. Liu, C. Gao, L. Feng, Y. Wei, Bio-inspired anti-oil-fouling chitosan-coated mesh for oil/water separation suitable for broad $\mathrm{pH}$ range and hyper-saline environments, ACS Appl. Mater. Interfaces 5 (2013) 11971-11976.

[S5] T. Li, J. Shen, Z. Zhang, S. Wang, D. Wei, A poly(2-(dimethylamino)ethyl methacrylate-co-methacrylic acid) complex induced route to fabricate a super-hydrophilic hydrogel and its controllable oil/water separation, RSC Adv. 6 (2016) 40656-40663.

[S6] S. Gao, J. Sun, P. Liu, F. Zhang, W. Zhang, S. Yuan, J. Li, J. Jin, A Robust polyionized hydrogel with an unprecedented underwater anti-crude-oil-adhesion property, Adv. Mater. 28 (2016) 5307-5314.

[S7] F. Lu, Y. Chen, N. Liu, Y. Cao, L. Xu, Y. Wei, L. Feng, A fast and convenient cellulose hydrogel-coated colander for high-efficiency oil-water separation, RSC Adv. 4 (2014) 32544-32548.

[S8] M. Su, Y. Liu, S. Li, Z. Fang, B. He, Y. Zhang, Y. Li, P. He, A rubber-like, underwater superoleophobic hydrogel for efficient oil/water separation, Chemical Engineering Journal, 361 (2019) 364-372 .

[S9] Y. Liu, M. Su, Y. Fu, P. Zhao, M. Xia, Y. Zhang, B. He, P. He, Corrosive environments tolerant, ductile and self-healing hydrogel for highly efficient oil/water separation, Chemical Engineering Journal, 354 (2018) 1185-1196.

[S10] W. Zhang, Y. Cao, N. Liu, Y. Chen, L. Feng, A novel solution-controlled hydrogel coated mesh for oil/water separation based on monolayer electrostatic self-assembly, RSC Adv. 4 (2014) 
51404-51410.

[S11] Z. Xue, S. Wang, L. Lin, L. Chen, M. Liu, L. Feng, L. Jiang, A novel superhydrophilic and underwater superoleophobic hydrogel-coated mesh for oil/water separation, Adv. Mater. 23 (2011) 4270-4273.

[S12] C. Teng, X. Lu, G. Ren, Y. Zhu, M. Wan, L. Jiang, Underwater self-cleaning PEDOT-PSS hydrogel mesh for effective separation of corrosive and hot oil/water mixtures, Adv. Mater. Interfaces. 1 (2015) 1400099. 
Movie S1

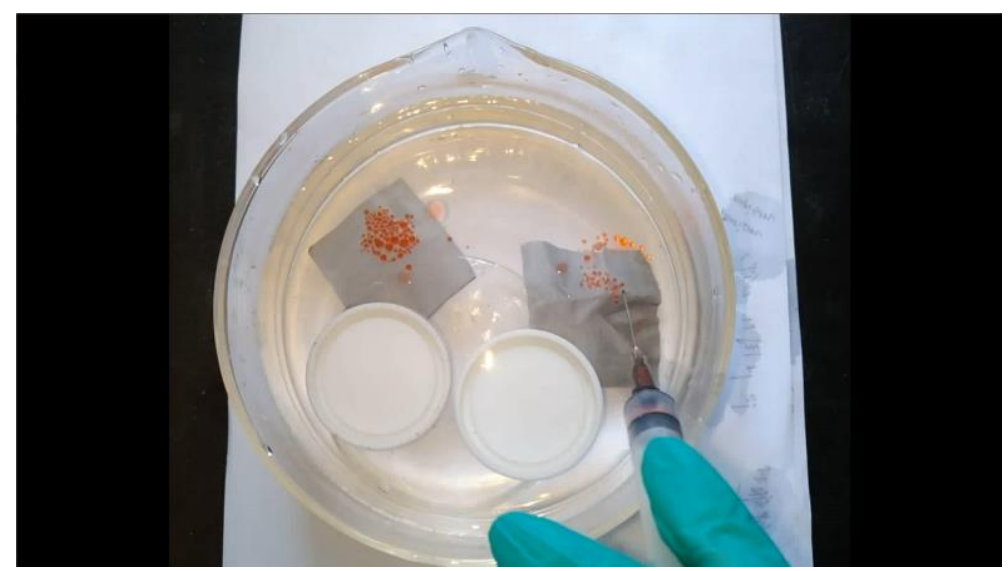

Movie S1 Underwater oil adhesion performance of the naked mesh and HEG-Mesh. 
Movie S2

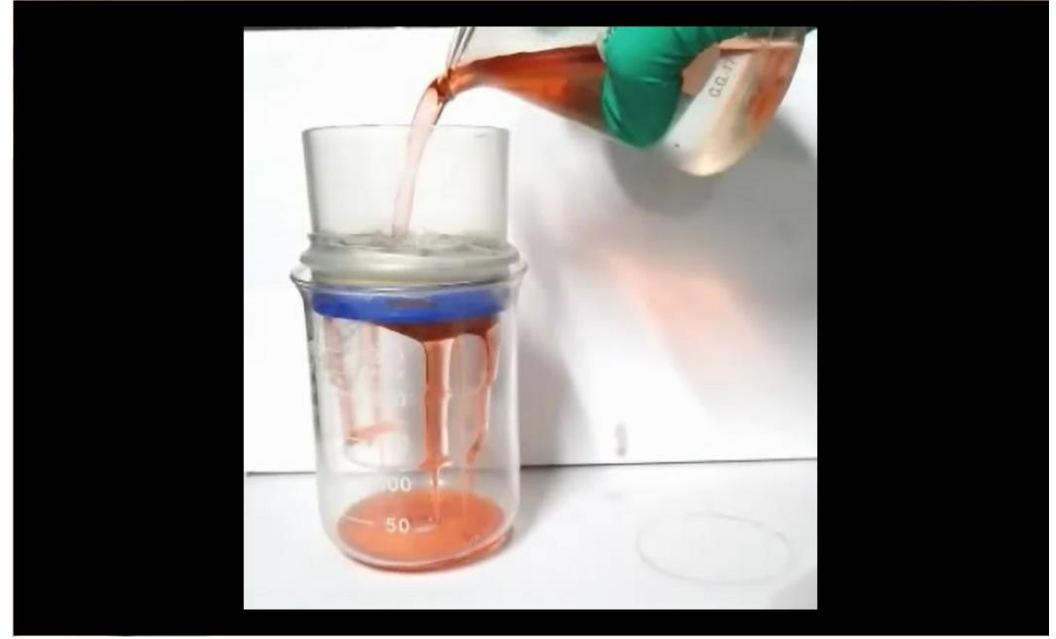

Movie S2 Oil-water separation process of the naked mesh only under gravity driving. 
Movie S3

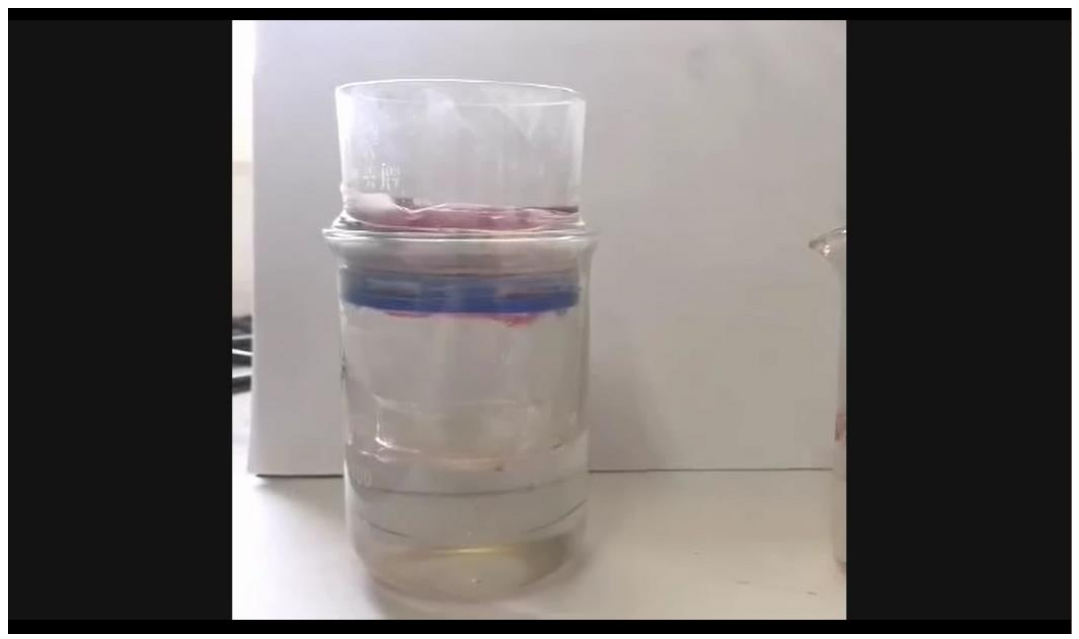

Movie S3 Oil-water separation process of the HEG-Mesh only under gravity driving. 